\title{
Effect of outcome measures on the apparent efficacy of ablation for atrial fibrillation: Why "success" is an inappropriate term
}

\author{
Nick Jackson ${ }^{1}$, Ehsan Mahmoodi ${ }^{2}$, James Leitch ${ }^{1}$, Malcolm Barlow ${ }^{1}$, Allan Davies ${ }^{1}$, \\ Nicholas Collins ${ }^{1}$, Lucy Leigh ${ }^{2}$, Christopher Oldmeadow ${ }^{2}$, and Andrew Boyle ${ }^{1}$ \\ ${ }^{1}$ John Hunter Hospital \\ ${ }^{2}$ The University of Newcastle
}

June 17, 2020

\begin{abstract}
INTRODUCTION: Different endpoint criteria, different durations of follow up and the completeness of follow up can dramatically affect the perceived benefits of Atrial Fibrillation (AF) ablation. METHODS: We defined three endpoints for recurrence of AF post ablation in a cohort of 200 patients with symptomatic AF, refractory to antiarrhythmic drugs (AADs). A "Strict Endpoint" where patients were considered to have a recurrence with any symptomatic or documented recurrence for [?]30 seconds with no blanking period, and off their AADs, a "Liberal Endpoint" where only documented recurrences after the blanking period, either on or off AADs were counted, and "Liberal Endpoint + Redo" which was the same as the Liberal endpoint but allowing for 1 redo ablation or 1 cardioversion (DCCV) during follow up. We also surveyed 50 patients on the waiting list for an AF ablation and asked them key questions regarding what they would consider to be a successful result for them. RESULTS: Freedom from recurrence of atrial tachyarrhythmias (AT) at 5 years was $62 \%$ for the strict endpoint, $73 \%$ for the liberal endpoint, and $80 \%$ for the liberal endpoint + redo group $(\mathrm{p}<0.001)$. Of the 50 patients surveyed awaiting AF ablation, $70 \%$ said they would still consider the procedure a success if it required one repeat ablation or one DCCV $(p=0.004)$, and $76 \%$ would be accepting of one or two recurrences during follow-up $(\mathrm{p}<0.001)$. CONCLUSION: In this study the most important predictor of AT recurrence following AF ablation was the criteria used to define recurrence.
\end{abstract}

\section{Introduction:}

The outcome of ablation for atrial fibrillation (AF) is often described in terms of success or failure, perhaps owing to the legacy from trials of surgical and then catheter-based ablation for pre-excitation. The paradigm of a "successful or unsuccessful" procedure for Wolf-Parkinson-White Syndrome is quite reasonable but much less so for more complex acquired conditions such as AF or ventricular tachycardia. These are chronic medical conditions influenced by many factors over time, quite apart from the technical aspects of an index procedure itself.

The principles of actuarial follow-up to measure efficacy for procedures such as coronary artery bypass surgery (CABG) have been well described for many years ${ }^{1}$ and consequently the efficacy of CABG is almost never described in terms of success or failure (or for that matter labelled as definitive or curative). Efficacy is dependent on a combination of factors including the hazard ratio for recurrence (based on clinical factors), data handling (should a survival or an event count model be used), censoring and most importantly followup duration and completeness. Ultimately efficacy is mostly a combination of the hazard ratio and the duration of follow-up and reducing this to success or failure is misleading. The nomenclature used in patient discussions can have a strong influence on the decisions patients make about their treatment so we should use terms that accurately reflect the data rather than emotive nomenclature such as success and failure.

The aim of this paper is to discuss how different patient populations and different criteria for the endpoint 
of the study can dramatically affect the perceived benefits and the outcomes in AF ablation trials. In this way we use our own previously published cohort of patients undergoing AF ablation to show how different the outcomes can appear with the same results and discuss what the patients themselves consider to be a successful outcome.

\section{Methods:}

Ethics for this study were obtained from the Hunter Research Ethics Committee (authorization number: AU201905-14). In order to perform this study, we analysed our cohort of 200 patients with symptomatic paroxysmal or persistent AF who had undergone an index cryoballoon ablation between the years of 2013 and 2016. This was a reanalysis of the study by Davies et al. 'Long term follow up of pulmonary vein isolation following cryoballoon ablation'.

All patients had symptomatic, paroxysmal or persistent AF and had failed treatment with at least one AAD. Patients were reviewed routinely at 3 months with a Holter monitor and a clinic visit. Then at 6,12 and 24 months with electrocardiograms and clinic visits. The follow up beyond this was at the discretion of the treating cardiologist. Patients were contacted at least twice by phone (beyond 24 months and again at the end of the follow up period) and all correspondence was reviewed from the local area electronic health record and the patient's treating cardiologist and general practitioner. Wherever a patient had symptoms consistent with recurrence further ECGs/telemetry were used to attempt to document this recurrence.

From this cohort we defined three different end points for ablation success in order to show how different the outcomes may seem despite the same procedures being performed on the same group of patients. For the Strict Outcome endpoint, we used the most strict criteria to define a successful outcome. For this endpoint a patient was deemed to have a recurrence if they had [?]30 seconds of symptomatic or asymptomatic atrial tachyarrhythmia, they were not allowed to be on any antiarrhythmic drugs beyond three months after ablation (recurrences during the usual 3 month blanking period were also counted) and patients were followed for 5 years. For the Liberal Outcome endpoint patients had to have a documented recurrence, were allowed a 3-month blanking period and were also allowed to be on antiarrhythmic medications during follow up. The Liberal Outcome + Redo endpoint was the same as the Liberal Outcome endpoint, however, they were allowed one repeat procedure (radiofrequency ablation) or one DCCV during their follow up as well. The Liberal Outcome and the Liberal Outcome + Redo endpoints were both evaluated for the success rates at 1 and 5 years.

The Strict Outcome endpoint was defined based on 2018 HRS consensus guidelines for follow up of patients in clinical trials with $\mathrm{AF}$ ablation ${ }^{3}$, however, we removed the blanking period as from a patient perspective a recurrence of $\mathrm{AF}$ is the same for them whether it occurs prior to 3 months or after. To come up with the criteria for the Liberal Outcome and the Liberal Outcome + Redo endpoints we surveyed 50 patients on the waiting list for an $\mathrm{AF}$ ablation and asked them key questions regarding what they would consider to be a successful result for them (see Tables 2 and 3). From the point of view of these questions, patients were then further divided into those with high burden of AF ([?] weekly symptoms from AF) or a low burden of AF (less than weekly).

\section{Primary Endpoint:}

The primary endpoint of the study was freedom from recurrence of symptomatic atrial tachyarrhythmia following pulmonary vein isolation according to the three endpoints as defined above. For the Strict Outcome endpoint any patient with symptoms beyond 30 seconds consistent with a recurrence was considered to have met this endpoint for the study.

\section{Statistical Analysis:}

All continuous variables are displayed as mean and standard deviation, and the categorical variables as count and percentage. Kaplan-Meier survival curves with an overall 5 years survival were used to depict time to recurrence of AF as per 3 different recurrence criteria. Frailty Cox Proportional Hazards models, using a gamma distribution for the shared frailty, were utilised to estimate pairwise Hazard Ratios between 
the three groups, and Cox $\mathrm{PH}$ regression models (with a gamma frailty) were utilised to determine the association between any of the predictors and the hazard of AF recurrence following AF ablation for all three endpoints. Student's t test or Mann-Whitney U test were used to compare the continuous variables based on the distribution, and the Chi squared test was used to compare categorical variables. All statistical analyses were programmed using R and SAS V9.4.

\section{Results:}

The baseline characteristics of the 200 patients undergoing cryoballoon ablation for drug refractory symptomatic atrial fibrillation are shown in Table 1.

Of the 50 consecutive patients who were interviewed as to what they would consider to be a successful outcome from ablation following pulmonary vein isolation, 26 patients $(52 \%)$ were considered high burden of symptoms and 24 low burden (48\%). The answers to the questions regarding patient expectations are shown in Tables 2 and 3. There were no statistically significant differences between the expectations of the outcome following AF ablation in the high versus low symptom burden groups.

The different endpoints used for freedom from recurrence of atrial tachyarrhythmias had a significant impact on outcome. Freedom from recurrence of atrial tachyarrhythmias as one year was $62 \%$ with Strict Outcomes, $87 \%$ with Liberal Outcomes and $90 \%$ with Liberal Outcomes + Redo (Frailty p-value $<0.005$ ). Freedom from recurrence of atrial tachyarrhythmias at five years was $62 \%$ with Strict Outcomes, $73 \%$ with Liberal Outcomes and $80 \%$ with Liberal Outcomes + Redo (Frailty p-value $<0.001$ ). Kaplan Meier survival curves for freedom from atrial tachyarrhythmia recurrence are shown in Figures 1 and 2. When performing pairwise comparisons between all three different endpoints, there was a statistically significant difference between the rates of atrial tachyarrhythmia free survival for all three different endpoints (Table 4).

The results of the adjusted frailty Cox $\mathrm{PH}$ regression model are reported in Table 5. The only significant predictor of freedom from atrial tachyarrhythmia recurrence in this model was the three different ways in which the endpoint was defined.

\section{Discussion:}

In this study the freedom from atrial tachyarrhythmia recurrence following pulmonary vein isolation ranged from $90 \%$ at 1 year (Liberal Outcomes + Redo) to $62 \%$ at five years (Strict Outcomes) according to the duration of follow up and how strict the criteria were for recurrence during that time period. In our Cox proportional hazards model the only significant predictors of freedom from AF were how the endpoint of recurrence was defined and not traditionally recognised predictors of success such as type of AF, age or LA size. Furthermore, when we surveyed 50 patients with AF who were awaiting pulmonary vein isolation we found that the majority of them would still consider ablation successful if it had to be repeated once (70\%), if one DCCV was required following ablation (70\%), or if they had just one or two recurrences of AF following their ablation procedure $(76 \%)$. However, the majority (68\%) of patients expected to be completely off AADs.

This study shows how subjective the notion of success can be following a procedure such as AF ablation and raises the important point of primarily considering the patients perspective when determining success or not. The HRS consensus statement for follow up of trials on AF ablation recommend the strict criteria of freedom from recurrence of any atrial tachyarrhythmia ([?]30 seconds) beyond a three month blanking period, off antiarrhythmic medications and with at least 3 monthly 24 hour Holter monitoring ${ }^{3}$. These criteria are important as they provide a consistent end point to allow for some comparison of different trials on different populations and with different techniques. Furthermore, more strict criteria ([?]30 sec of atrial tachyarrhythmia) will generally mean a greater number of patients reach the study endpoint and increases the statistical power of the studies. For this reason, it is important to keep this clear definition, but at the same time discuss what this means in clinical practice. For example, many patients may consider a one-year measure of efficacy too short or feel misled if one-year freedom from recurrence is described as a success rate.

In the 2009 meta-analysis of trials of AF ablation versus AAD therapy the 1-year freedom from recurrence 
of atrial fibrillation ranged from $57 \%$ (over 2,800 patients) with a single procedure to $71 \%$ with multiple procedures (over 3,481 patients) and $77 \%$ with multiple procedures on AADs ${ }^{4}$. Freedom from recurrent AF at one year on AADs alone in this meta-analysis was $52 \%{ }^{4}$. In these studies, the frequency and duration of Holter monitoring is less important since the aim is to compare two different treatments. It is more important that it is equivalent in each arm and sufficient to detect the expected differences in efficacy between the two groups. These trials primarily used ECGs at 3 monthly follow up visits to look for AF recurrence, with Holter or event monitors used in fewer than half of the ablation studies and fewer than a quarter of the AAD studies. These trials established the fact that PVI was superior to AAD therapy in reducing the time to recurrent $\mathrm{AF}$ in patients who had already failed an AAD. More recent trials, however, have used implantable cardiac monitors to determine exactly what the recurrence rates of AF are after ablation.

The Circa-dose study ${ }^{5}$ highlighted the importance of monitoring in defining PVI success by showing that following cryoballoon or radiofrequency PVI, the freedom from any symptomatic recurrence of atrial tachyarrhythmia was $73-79 \%$, however, the freedom from any recurrence [?]30 seconds of atrial tachyarrhythmia as detected by ILR was $52-54 \% .^{5}$ This finding raises the question of the impact of a placebo affect from ablation therapy but also questions the clinical relevance of very short duration episodes of AF that are not symptomatic. Further to this, in a study by Steinberg et al. ${ }^{6}$ prospectively looking at episodes of pacemaker detected AF they found that shorter index episodes of $\mathrm{AF}$ ( $<3.8$ hours duration) did not predict recurrence of clinically meaningful AF episodes over the subsequent 3.7 year duration of the study.

The results of the Circa-dose study ${ }^{5}$ could be interpreted to say that AF ablation was unsuccessful in up to $48 \%$ of patients at 1 year following an $\mathrm{AF}$ ablation. However, both the Circa-dose ${ }^{5}$ and the Close to Cure $^{7}$ studies also compared the mean burden of AF on ILR prior to and following ablation and this showed a 98-100\% mean reduction in AF burden with ablation. Prior to ablation, however, patients were in AF for a median of $1.5-4 \%$ of the time and following ablation they were is AF for a median of $0.00 \%$ of the time. In the CAPTAF study ${ }^{8}$ which included patients with persistent AF as well, the median burden of AF was reduced from $24.9 \%$ to $5.5 \%$ with AF ablation ( $78 \%$ reduction). Median burden reduction speaks to the strong efficacy of PVI for reducing the burden of AF in individual patients. In the Close to Cure study ${ }^{7}$ almost all patients had a lower burden of AF following ablation and even those patients with some residual AF still reported improved QoL scores on the basis of this reduced AF burden. The authors of this study point out that AF burden may be a more meaningful endpoint for AF studies as AF burden correlates with symptoms and risk of stroke and heart failure ${ }^{9-11}$. Since QoL improves in patients post ablation who are free from or have reduced $\mathrm{AF}$ burden ${ }^{12,13}$ this may be a more representative endpoint than simply a survival analysis that sensors patients after a single brief recurrence.

AF ablation trials have long used a 3-month blanking period following ablation before counting recurrent episodes towards the trial endpoint. This is chiefly since AF ablation may lead to inflammation, transient autonomic imbalance or delayed lesion maturation and a period of time for atrial remodelling may also be important in success following ablation ${ }^{14,15}$. Some trials have even allowed redo procedures within this blanking period, suggesting some early recurrence is due to pulmonary vein reconnection ${ }^{16,17}$. Whilst this 3-month blanking period may more fairly show the efficacy of AF ablation when assessed by time to first recurrence, from a patient's perspective, if they have a recurrence, require a cardioversion or a repeat procedure it is irrelevant at what time this occurs. Furthermore, some studies have suggested that recurrences beyond a 1-month blanking period are predictive of recurrence ${ }^{18}$ and this may be different for different PVI techniques ${ }^{19}$. For this reason assessing burden pre and post ablation without a 3-month blanking period may give a more accurate assessment of clinical efficacy since a patient with a low burden of paroxysmal AF pre ablation, who has a significant 'flare up' in their AF symptoms in the first three months following ablation may not consider their procedure a success.

A number of clinical factors have long been known to influence the success of AF ablation. The most important of these may be the presence of persistent AF, female sex, renal dysfunction, low ejection fraction, advanced age and larger left atrial size ${ }^{20-23}$. These factors have been incorporated into various scores such as the APPLE, ATLAS, DR-FLASH and MB-LATER scores that could give a patient a percentage likelihood 
that they would be free from any AF at one year between 19\% to 71\%. Although these scores were developed to predict recurrence at difference time points and despite both the APPLE and MB-LATER scores being the only ones validated in external cohorts, both these scores still had modest predictive capacity on ROC analysis (AUC 0.64 and 0.65 for APPLE and MB LATER respectively) ${ }^{24}$. The number of different scores developed, however, demonstrates the differences in predictive variables in different populations and these predictions have wide confidence intervals for individual cases. Furthermore these scores do not take into account other potentially important factors such as the experience of the operator ${ }^{25}$ or the willingness of the patient to address modifiable risk factors around the time of and following ablation ${ }^{26}$.

The success of AF ablation is further dependent upon the indication for which the ablation is being performed. Much of the discussion to this point has focussed on AF ablation to reduce symptoms directly related to episodes of $\mathrm{AF}$, however, a number of randomised controlled trials have shown an improvement in ejection fraction and/or a reduction in mortality in patients with severe systolic impairment and $\mathrm{AF}^{27,28,29,30}$. In the largest of these RCTs the CASTLE-AF ${ }^{27}$ study the NNT to save one life was 8.6. In these studies looking at ablation to treat heart failure, patients have primarily had implantable defibrillators and these have shown a substantial reduction in the burden of AF despite the presence of structural heart disease. In these studies, low residual burdens of AF were still associated with improved heart failure outcomes. These patients were also much more commonly continued on AADs beyond a three-month blanking period, their AF was more commonly persistent or chronic persistent and they were less likely to be aware of individual episodes of AF. For these reasons the success of an AF ablation here cannot be assessed in terms of conventional endpoints but rather from heart failure endpoints.

\section{Limitations:}

In our cohort we primarily assessed the recurrence of AF by the presence of symptoms and our rates of AF recurrence would have been higher with more frequent monitoring or implantable loop recorders. The purpose of this study, however, was not to precisely define the incidence of recurrent AF following ablation but to demonstrate how reliant the success rates of AF ablation procedures are upon study design.

\section{Conclusion:}

In this study the most important predictor of atrial fibrillation recurrence following ablation was the criteria used to define recurrence (duration of follow up, the presence of a blanking period and whether AADs were allowed or not). We showed that the majority of patients still considered AF ablation a successful treatment if they had up to two recurrences of AF, one repeat procedure or one DCCV. This study highlights the complexity in understanding the 'success' of a procedure such as AF ablation and emphasizes the importance of a patient centred prospective.

\section{References:}

1. Carr BM, Romeiser J, Ruan J, et al. Long-Term Post-CABG Survival: Performance of Clinical Risk Models Versus Actuarial Predictions.J Card Surg. 2016;31(1):23-30.

2. Davies AJ, Jackson N, Barlow M, Leitch J. Long Term Follow-up of Pulmonary Vein Isolation Using Cryoballoon Ablation. Heart Lung Circ. 2016;25(3):290-295.

3. Calkins H, Hindricks G, Cappato R, et al. 2017 HRS/EHRA/ECAS/APHRS/SOLAECE expert consensus statement on catheter and surgical ablation of atrial fibrillation. Europace : European pacing, arrhythmias, and cardiac electrophysiology : journal of the working groups on cardiac pacing, arrhythmias, and cardiac cellular electrophysiology of the European Society of Cardiology.2018;20(1):e1-e160.

4. Calkins H, Reynolds MR, Spector P, et al. Treatment of atrial fibrillation with antiarrhythmic drugs or radiofrequency ablation: two systematic literature reviews and meta-analyses. Circulation Arrhythmia and electrophysiology. 2009;2(4):349-361.

5. Andrade JG, Champagne J, Dubuc M, et al. Cryoballoon or Radiofrequency Ablation for Atrial Fibrillation Assessed by Continuous Monitoring: A Randomized Clinical Trial. Circulation.2019;140(22):1779-1788. 
6. Steinberg JS, O'Connell H, Li S, Ziegler PD. Thirty-Second Gold Standard Definition of Atrial Fibrillation and Its Relationship With Subsequent Arrhythmia Patterns: Analysis of a Large Prospective Device Database. Circulation Arrhythmia and electrophysiology.2018;11(7):e006274.

7. Duytschaever M, De Pooter J, Demolder A, et al. Long-term impact of catheter ablation on arrhythmia burden in low-risk patients with paroxysmal atrial fibrillation: The CLOSE to CURE study. Heart rhythm : the official journal of the Heart Rhythm Society. 2019.

8. Blomstrom-Lundqvist C, Gizurarson S, Schwieler J, et al. Effect of Catheter Ablation vs Antiarrhythmic Medication on Quality of Life in Patients With Atrial Fibrillation: The CAPTAF Randomized Clinical Trial.JAMA. 2019;321(11):1059-1068.

9. Bjorkenheim A, Brandes A, Magnuson A, Chemnitz A, Edvardsson N, Poci D. Patient-Reported Outcomes in Relation to Continuously Monitored Rhythm Before and During 2 Years After Atrial Fibrillation Ablation Using a Disease-Specific and a Generic Instrument. J Am Heart Assoc. 2018;7(5).

10. Glotzer TV, Daoud EG, Wyse DG, et al. The relationship between daily atrial tachyarrhythmia burden from implantable device diagnostics and stroke risk: the TRENDS study. Circulation Arrhythmia and electrophysiology. 2009;2(5):474-480.

11. Go AS, Reynolds K, Yang J, et al. Association of Burden of Atrial Fibrillation With Risk of Ischemic Stroke in Adults With Paroxysmal Atrial Fibrillation: The KP-RHYTHM Study. JAMA Cardiol.2018;3(7):601-608.

12. Packer DL, Kowal RC, Wheelan KR, et al. Cryoballoon ablation of pulmonary veins for paroxysmal atrial fibrillation: first results of the North American Arctic Front (STOP AF) pivotal trial. Journal of the American College of Cardiology. 2013;61(16):1713-1723.

13. Wilber DJ, Pappone C, Neuzil P, et al. Comparison of antiarrhythmic drug therapy and radiofrequency catheter ablation in patients with paroxysmal atrial fibrillation: a randomized controlled trial. JAMA. 2010;303(4):333-340.

14. Andrade JG, Macle L, Khairy P, et al. Incidence and significance of early recurrences associated with different ablation strategies for AF: a STAR-AF substudy. Journal of cardiovascular electrophysiology.2012;23(12):1295-1301.

15. Andrade J, Khairy P, Dobrev D, Nattel S. The clinical profile and pathophysiology of atrial fibrillation: relationships among clinical features, epidemiology, and mechanisms. Circulation research.2014;114(9):14531468.

16. Das M, Wynn GJ, Saeed Y, et al. Pulmonary Vein Re-Isolation as a Routine Strategy Regardless of Symptoms: The PRESSURE Randomized Controlled Trial. JACC Clin Electrophysiol. 2017;3(6):602-611.

17. Mujovic N, Marinkovic M, Markovic N, et al. The relationship of early recurrence of atrial fibrillation and the 3-month integrity of the ablation lesion set. Sci Rep. 2018;8(1):9875.

18. Jackson N, Barlow M, Leitch J, Attia J. Treating atrial fibrillation: pulmonary vein isolation with the cryoballoon technique. Heart Lung Circ. 2012;21(8):427-432.

19. Kuck KH, Furnkranz A, Chun KR, et al. Cryoballoon or radiofrequency ablation for symptomatic paroxysmal atrial fibrillation: reintervention, rehospitalization, and quality-of-life outcomes in the FIRE AND ICE trial. Eur Heart J. 2016;37(38):2858-2865.

20. Kornej J, Hindricks G, Shoemaker MB, et al. The APPLE score: a novel and simple score for the prediction of rhythm outcomes after catheter ablation of atrial fibrillation. Clinical research in cardiology : official journal of the German Cardiac Society. 2015;104(10):871-876.

21. Mujovic N, Marinkovic M, Markovic N, Shantsila A, Lip GY, Potpara TS. Prediction of very late arrhythmia recurrence after radiofrequency catheter ablation of atrial fibrillation: The MB-LATER clinical 
score.Sci Rep. 2017;7:40828.

22. Kosiuk J, Dinov B, Kornej J, et al. Prospective, multicenter validation of a clinical risk score for left atrial arrhythmogenic substrate based on voltage analysis: DR-FLASH score. Heart rhythm : the official journal of the Heart Rhythm Society.2015;12(11):2207-2212.

23. Mesquita J, Ferreira AM, Cavaco D, et al. Development and validation of a risk score for predicting atrial fibrillation recurrence after a first catheter ablation procedure - ATLAS score. Europace : European pacing, arrhythmias, and cardiac electrophysiology : journal of the working groups on cardiac pacing, arrhythmias, and cardiac cellular electrophysiology of the European Society of Cardiology.2018;20(FI_3):f428-f435.

24. Kornej J, Schumacher K, Zeynalova S, et al. Time-dependent prediction of arrhythmia recurrences during long-term follow-up in patients undergoing catheter ablation of atrial fibrillation: The Leipzig Heart Center AF Ablation Registry. Sci Rep.2019;9(1):7112.

25. Sairaku A, Yoshida Y, Nakano Y, et al. Who is the operator, that is the question: a multicentre study of catheter ablation of atrial fibrillation. Europace : European pacing, arrhythmias, and cardiac electrophysiology : journal of the working groups on cardiac pacing, arrhythmias, and cardiac cellular electrophysiology of the European Society of Cardiology. 2016;18(9):1352-1356.

26. Pathak RK, Middeldorp ME, Lau DH, et al. Aggressive risk factor reduction study for atrial fibrillation and implications for the outcome of ablation: the ARREST-AF cohort study. Journal of the American College of Cardiology. 2014;64(21):2222-2231.

27. Marrouche NF, Brachmann J, Andresen D, et al. Catheter Ablation for Atrial Fibrillation with Heart Failure. The New England journal of medicine. 2018;378(5):417-427.

28. Prabhu S, Taylor AJ, Costello BT, et al. Catheter Ablation Versus Medical Rate Control in Atrial Fibrillation and Systolic Dysfunction: The CAMERA-MRI Study. Journal of the American College of Cardiology. 2017;70(16):1949-1961.

29. Hunter RJ, Berriman TJ, Diab I, et al. A randomized controlled trial of catheter ablation versus medical treatment of atrial fibrillation in heart failure (the CAMTAF trial). Circulation Arrhythmia and electrophysiology. 2014;7(1):31-38.

30. Di Biase L, Mohanty P, Mohanty S, et al. Ablation Versus Amiodarone for Treatment of Persistent Atrial Fibrillation in Patients With Congestive Heart Failure and an Implanted Device: Results From the AATAC Multicenter Randomized Trial. Circulation.2016;133(17):1637-1644.

Table 1. Baseline characteristics (total number $=200$ )

\begin{tabular}{ll}
\hline Variable & Number \\
\hline Age, mean (SD) & $61.7(10)$ \\
Female sex, number (\%) & $76(38)$ \\
Left atrial dimension, mm, mean (SD) & $40.5(6.6)$ \\
Left ventricle ejection fraction (\%), mean (SD) & $61.3(8.8)$ \\
Paroxysmal AF, number (\%) & $138(69)$ \\
Persistent AF, number (\%) & $61(31)$ \\
Symptom duration (months) & $36(23-60)$ \\
Congestive cardiac failure, number (\%) & $27(14)$ \\
Diabetes mellitus, number (\%) & $15(7.5)$ \\
Hypertension, number (\%) & $106(53)$ \\
Stroke/TIA, number (\%) & $8(4)$ \\
Pre-Ablation Anti-arrhythmic drugs & \\
Class Ic (Flecainide), number (\%) & $56(28.8 \%)$ \\
Class III (Sotalol or Amiodarone), number (\%) & $134(67.7 \%)$
\end{tabular}




\begin{tabular}{ll}
\hline Variable & Number \\
\hline Oral Anticoagulation & $180(90 \%)$ \\
\hline
\end{tabular}

Table 2. The Results of 50 patents with AF awaiting ablation who were surveyed at to what they would consider acceptable in relation to outcomes from the procedure.

$\mathrm{AAD}=$ Antiarrhythmic drugs. DCCV $=$ Direct current cardioversion.

\begin{tabular}{|c|c|c|c|}
\hline QUESTION & Yes, Count $(\%)$ & No, Count $(\%)$ & P-Value \\
\hline $\begin{array}{l}\text { High burden versus low } \\
\text { burden AF. (High: } \\
\text { weekly } 1 \text {, Low < weekly } \\
0 \text { ) }\end{array}$ & $26(52)$ & $24(48)$ & 0.77 \\
\hline $\begin{array}{l}\text { Would you consider } \\
\text { having just one or two } \\
\text { recurrences a good } \\
\text { outcome? }\end{array}$ & $38(76)$ & $12(24)$ & $<0.001$ \\
\hline $\begin{array}{l}\text { Would you be } \\
\text { accepting one repeat } \\
\text { procedure or one } \\
\text { DCCV? }\end{array}$ & $35(70)$ & $15(30)$ & 0.004 \\
\hline $\begin{array}{l}\text { Would you consider } \\
\text { having half the amount } \\
\text { of AF you previously } \\
\text { had as a Successful } \\
\text { procedure/good } \\
\text { outcome? (half the } \\
\text { duration and/or half } \\
\text { the number of } \\
\text { episodes) }\end{array}$ & $35(70)$ & $15(30)$ & 0.004 \\
\hline $\begin{array}{l}\text { Would you expect to be } \\
\text { completely off AADs? }\end{array}$ & $34(68)$ & $16(32)$ & 0.01 \\
\hline
\end{tabular}

Table 3. The Results of 50 patents with AF awaiting ablation who were surveyed as to what they would consider acceptable in relation to outcomes from the procedure.

$\mathrm{AAD}=$ Antiarrhythmic drugs. DCCV $=$ Direct current cardioversion.

\section{QUESTION}

Q1 - would you consider having just one or two recurrences a good outcome?

Q2 - Would you be accepting of one repeat procedure or one DCCV?

Q3 - Would you consider halving the duration/number of AF a successful/good outcome?

Q4 - Would you expect to be completely off AADs?

\begin{tabular}{ll}
\hline Response & High Burden (1 \\
No & $6(23.1)$ \\
Yes & $20(76.9)$ \\
No & $7(26.9)$ \\
Yes & $19(73.1)$ \\
No & $9(34.6)$ \\
Yes & $17(65.4)$ \\
No & $9(34.6)$ \\
Yes & $17(65.4)$ \\
\hline
\end{tabular}

Figure 1 shows the survival free from AT recurrence according to the three specified endpoints of Strict 
Outcome, Liberal Outcome and Liberal Outcomes + Redo out to one year.

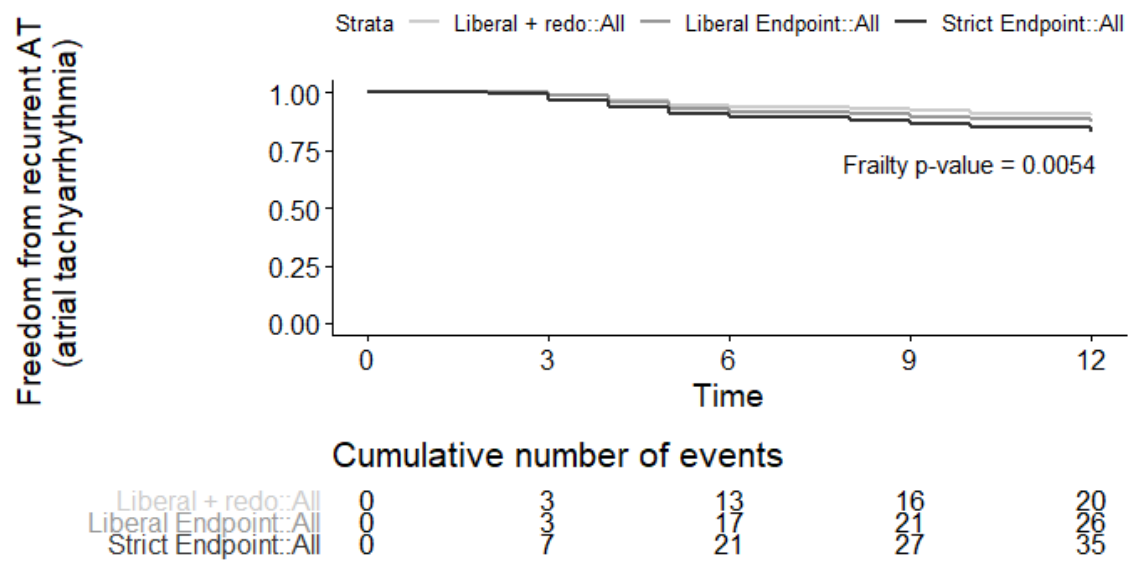

Figure 2 shows the survival free from AT recurrence according to the three specified endpoints of Strict Outcome, Liberal Outcome and Liberal Outcomes + Redo out to five years.

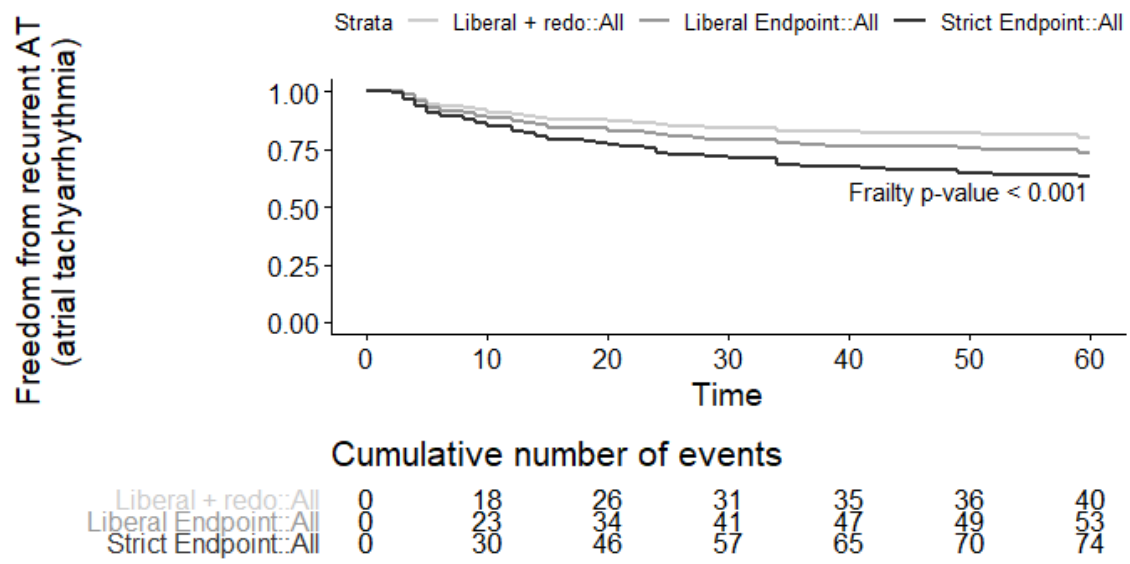

Table 4. shows the pairwise comparisons between each of the defined end point groups.

\begin{tabular}{lll}
\hline Comparison & HR (95\% CI) & P-value \\
Group 3 (Loose + redo) vs 2 (Loose) & $0.53(0.34,0.82)$ & $<0.01$ \\
Group 3 (Loose + redo) vs 1 (Strict) & $0.31(0.20,0.48)$ & $<0.01$ \\
Group 2 (Loose) vs 1 (Strict) & $0.59(0.41,0.85)$ & $<0.01$ \\
\hline
\end{tabular}

Table 5. shows Hazard Ratios from Univariable Cox Proportional Hazards Models^, and multivariable Frailty Cox Proportional Hazards Regression Model

\begin{tabular}{llllll}
\hline & & Univariable & Univariable & Multivariable & Multivariable \\
Variable & HR (95\% CI) & P-value & HR (95\% CI) & P-value \\
Group & Liberal +Redo vs Strict & $0.31(0.20,0.48)$ & $<.0001$ & $0.35(0.22,0.56)$ & $<0.001$
\end{tabular}




\begin{tabular}{llllll} 
& Liberal vs Strict & $0.59(0.41,0.85)$ & 0.004 & $0.65(0.43,0.97)$ & 0.034 \\
& Liberal + Redo vs Liberal & $0.53(0.34,0.82)$ & 0.004 & $0.57(0.35,0.91)$ & 0.02 \\
Age & & $1.03(0.99,1.06)$ & 0.12 & $1.03(0.97,1.10)$ & 0.33 \\
LA Dimension & & $1.00(0.95,1.05)$ & 0.97 & $0.97(0.86,1.09)$ & 0.61 \\
LVEF & $1.00(0.97,1.04)$ & 0.86 & $0.99(0.90,1.09)$ & 0.87 \\
Sex & Female & $2.42(1.29,4.53)$ & 0.005 & $3.10(0.78,12.26)$ & 0.10 \\
AF Type & paroxysmal & $0.60(0.32,1.14)$ & 0.11 & $0.56(0.13,2.45)$ & 0.44 \\
Hypertension & Yes & $1.36(0.72,2.56)$ & 0.33 & $2.07(0.58,7.33)$ & 0.26 \\
Diabetes Mellitus & Yes & $0.30(0.04,2.19)$ & 0.23 & $1.09(0.09,13.89)$ & 0.94 \\
\hline
\end{tabular}

